\title{
Modulation of Osteoblastic Cell Efferocytosis by Bone Marrow Macrophages
}

\author{
Megan N. Michalski, ${ }^{1}$ Amy J. Koh, ${ }^{1}$ Savannah Weidner, ${ }^{1}$ Hernan Roca, ${ }^{1}$ \\ and Laurie K. McCauley ${ }^{1,2 *}$ \\ ${ }^{1}$ Department of Periodontics and Oral Medicine, University of Michigan School of Dentistry, Ann Arbor, \\ Michigan 48109 \\ ${ }^{2}$ Department of Pathology, University of Michigan Medical School, Ann Arbor, Michigan 48109
}

\begin{abstract}
Apoptosis occurs at an extraordinary rate in the human body and the effective clearance of dead cells (efferocytosis) is necessary to maintain homeostasis and promote healing, yet the contribution and impact of this process in bone is unclear. Bone formation requires that bone marrow stromal cells (BMSCs) differentiate into osteoblasts which direct matrix formation and either become osteocytes, bone lining cells, or undergo apoptosis. A series of experiments were performed to identify the regulators and consequences of macrophage efferocytosis of apoptotic BMSCs (apBMSCs). Bone marrow derived macrophages treated with the anti-inflammatory cytokine interleukin-10 (IL-10) exhibited increased efferocytosis of apBMSCs compared to vehicle treated macrophages. Additionally, IL-10 increased anti-inflammatory M2-like macrophages $\left(\mathrm{CD} 206^{+}\right)$, and further enhanced efferocytosis within the CD206 ${ }^{+}$population. Stattic, an inhibitor of STAT3 phosphorylation, reduced the IL-10-mediated shift in M2 macrophage polarization and diminished IL-10-directed efferocytosis of apBMSCs by macrophages implicating the STAT3 signaling pathway. Cell culture supernatants and RNA from macrophages co-cultured with apoptotic bone cells showed increased secretion of monocyte chemotactic protein 1/chemokine (C-C motif) ligand 2 (MCP-1/CCL2) and transforming growth factor beta 1 (TGF- $\beta 1$ ) and increased ccl2 gene expression. In conclusion, IL-10 increases M2 macrophage polarization and enhances macrophage-mediated engulfment of apBMSCs in a STAT3 phosphorylation-dependent manner. After engulfment of apoptotic bone cells, macrophages secrete TGF- $\beta 1$ and MCP-1/CCL2, factors which fuel the remodeling process. A better understanding of the role of macrophage efferocytosis as it relates to normal and abnormal bone turnover will provide vital information for future therapeutic approaches to treat bone related diseases. J. Cell. Biochem. 117: 2697-2706, 2016. @ 2016 Wiley Periodicals, Inc.
\end{abstract}

KEY WORDS: APOPTOSIS; BONE BIOLOGY; EFFEROCYTOSIS; MACROPHAGES; CYTOKINES; OSTEOBLASTS

$\mathrm{T}$ he critical process of bone formation depends on the lifespan and activity of osteoblasts lining the bone surface. Three fates have been described for osteoblasts: they either become osteocytes embedded in mineralized matrix, bone-lining cells which form layers over bone surfaces and at remodeling sites, or they undergo programmed cell death (apoptosis). Of the osteoblasts initially at remodeling sites, 30-50\% become osteocytes and bone-lining cells, which leaves a large percentage of cells thought to undergo apoptosis [Jilka et al., 1998]. The course of events after osteoblast apoptosis has been under appreciated. This is in part due to the inability of assays to accurately detect and quantify apoptotic osteoblasts readily undergoing apoptosis. In normal physiology, cell death is followed by rapid and efficient removal of apoptotic cells by phagocytic cells, predominantly macrophages. This process of apoptotic cell recognition and clearance is termed efferocytosis [Ravichandran and Lorenz, 2007].

Macrophages are immune cells known for their role in infection and inflammation. A focus on their role in bone has only recently emerged [Sinder et al., 2015]. Macrophages are prominent players in bone homeostasis and are highly implicated in fracture healing [Chang et al., 2008; Alexander et al., 2011; Cho et al., 2014]. They are often found at sites of remodeling, and are intimately associated with bone forming osteoblastic cells. Additionally, macrophage numbers increase significantly in fracture sites, and bone repair is severely diminished after tibial injury in macrophage-ablated mouse models [Alexander et al., 2011]. Apoptotic cells increase in sites of injury,

Grant sponsor: National Institutes of Health, National Institute of Diabetes, and Digestive and Kidney Diseases; Grant number: R01DK053904 ; Grant sponsor: National Institutes of Health, National Institute of Dental, and Craniofacial Research; Grant numbers: F30DE025154, T32DE007057.

*Correspondence to: Laurie K. McCauley, DDS, PhD, University of Michigan, School of Dentistry, 1011 N. University Ave, Ann Arbor, MI 48109-1078. E-mail: mccauley@umich.edu

Manuscript Received: 4 April 2016; Manuscript Accepted: 5 April 2016

Accepted manuscript online in Wiley Online Library (wileyonlinelibrary.com): 7 April 2016

DOI 10.1002/jcb.25567 • @ 2016 Wiley Periodicals, Inc. 
and a crucial component of the healing process is the effective clearance of these cells by phagocytes, including macrophages. The recognition and subsequent efferocytosis of dead and dying cells leads to the secretion of anti-inflammatory cytokines such as TGF- $\beta$ and IL-10 [Voll et al., 1997; Fadok et al., 1998], as well as osteoinductive factors including osteopontin, and BMP-2 [Champagne et al., 2002; Takahashi et al., 2004; Honda et al., 2006]. The process of efferocytosis has been extensively studied in other tissues. Recently, macrophages were found to efferocytose apoptotic osteoblasts [McCauley et al., 2014] yet the impact of efferocytosis in bone has not been clearly defined.

Macrophages have been shown to polarize into two populations: classically activated M1 macrophages and alternatively activated M2 macrophages. M2 macrophages are present during the resolution phase of inflammation and are responsible for antiinflammatory cytokine production and enhanced efferocytosis [Xu et al., 2006; Bystrom et al., 2008]. In the presence of the anti-inflammatory cytokines IL-4 and IL-10, macrophages polarize to M2-like macrophages [Mantovani et al., 2004], and increase apoptotic lymphocyte and apoptotic neutrophil clearance [Ogden et al., 2005; Lingnau et al., 2007]. IL-10 is an important bone cytokine which inhibits osteoclastogenesis [Xu et al., 1995] and exhibits a protective role in periodontal disease [Moretti et al., 2015]. Furthermore, IL-10 deficient mice exhibit low bone mass phenotypes with reduced bone formation compared to wild-type controls [Xu et al., 1995; Dresner-Pollak et al., 2004]. To our knowledge, the role of IL-10-induced efferocytosis in bone has not been investigated.

Additonally, it is unclear which factors regulate the clearance of apoptotic bone cells by bone resident macrophages and how macrophages respond to the englulfment of an apoptotic bone cell. The apoptotic cell identity can impact the response elicited by the phagocyte and the response of a bone marrow macrophage to an apoptotic bone cell is of interest to the present work. This study aimed to determine factors and signaling which affect macrophage efferocytosis of apoptotic osteoblastic cells and changes in macrophage gene expression in response to these apoptotic cells.

\section{MATERIALS/METHODS}

\section{ANIMALS}

All animals were maintained in accordance with institutional animal care and use guidelines, and experimental protocols were approved by the Institutional Animal Care and Use Committee of the University of Michigan. C57BL/6J (The Jackson Laboratory, Bar Harbor, ME) mice were used for in vitro experiments unless otherwise indicated. Human CD68-GFP reporter mice (hCD68-GFP) mice were generously provided by Dr. Ajay Chawla (University of California San Francisco). hCD68-GFP mice were generated by cloning a cDNA fragment encoding EGFP (from pEGFP-N1 vector, Invitrogen) into the 1,265 vector containing human CD68 promoter $(-2.9 \mathrm{~kb})$. DNA was then excised from the vector and injected into mouse oocytes by pro-nuclear injection [Iqbal et al., 2014].

\section{CELL CULTURE}

Primary bone marrow cells were collected from 4- to 8-week old C57BL/6J or hCD68-GFP mice. Bone marrow-derived macrophages (BMMs) were differentiated in vitro from bone marrow flush in $\alpha$-MEM medium (10\% FBS, Pen/Strep, glutamine) with murine M-CSF (30 ng/mL eBioscience) for 6 days. At day 7, macrophages were plated at $2.5 \times 10^{5}$ cells/well in 12-well plates (for efferocytosis assays) or $1.5 \times 10^{6}$ cells/well in 6 -well plates (for protein/RNA). Bone marrow stromal cells (BMSCs) were derived from bone marrow flush and cultured in $\alpha$-MEM medium (20\% FBS, Pen/Strep, glutamine) containing $10 \mathrm{nM}$ dexamethasone (Sigma) and used at passage 1-2. Bone marrow neutrophils were isolated as previously described [Swamydas and Lionakis, 2013]. Briefly, bone marrow was flushed from 8- to 12-week old C57BL/6J mice with RPMI supplemented with 10\% FBS and 2 mM EDTA, red blood cells lysed using $0.2 \% \mathrm{NaCl}$, and neutrophils separated by density gradient centrifugation (Histopaque 1119 and Histopaque 1077). Neutrophils were harvested at the interface of the Histopaque 1119 and Histopaque 1077 layers and confirmed using FACs anaylsis for CD11b ${ }^{+} \mathrm{Ly6G}^{+}$cells.

\section{MACROPHAGE TREATMENT}

All recombinant murine proteins were obtained from RetD Systems. BMMs were treated at day 8 with $\mathrm{rmIL}-10(0.1-100 \mathrm{ng} / \mathrm{mL})$, rmCCL2/MCP-1 (10 ng/mL), rmMFG-E8 $\quad(10 \mathrm{ng} / \mathrm{mL}), \quad$ sIL-6R $(100 \mathrm{ng} / \mathrm{mL})$, or vehicle $(0.5 \% \mathrm{BSA}$ in $1 \mathrm{X} \mathrm{PBS})$ for $4-24 \mathrm{~h}$ in low serum containing media (0.5\% FBS). In a set of experiments BMMs were pre-treated with an inhibitor of pSTAT3 (Stattic, $6.25 \mu \mathrm{M}$, CalBiochem) or DMSO (vehicle) $2 \mathrm{~h}$ prior to rmIL-10 treatment.

\section{EFFEROCYTOSIS ASSAY}

BMSCs were stained with CellTracker Deep Red (APC ${ }^{+}$, Invitrogen) and exposed to UV light for $30 \mathrm{~min}$ to induce apoptosis. Apoptotic BMSCs (apBMSCs) were recovered for $2 \mathrm{~h}$ at $37^{\circ} \mathrm{C}$, enumerated via trypan blue exclusion (confirming cell death), and added to BMM cultures at a 1:1 ratio for 0.5-6 h. BMMs co-cultured with apBMSCs were harvested and stained with F4/80-FITC (Abd Serotec, CI:A3-1), fixed with $1 \%$ formalin and efferocytosis was assessed via flow cytometric (FACs) analyses (BD FACSAria ${ }^{\mathrm{TM}}$ III) for double labeled cells $\left(\mathrm{FITC}^{+} \mathrm{APC}^{+}\right)$reflecting engulfment. Macrophages cultured alone were harvested and stained with the following macrophagespecific antibodies: F4/80 FITC (CI:A3-1, BIORAD), CD86 PE (GL-1, Biolegend), and CD206 PE (C068C2, Biolegend). Cells were fixed, permeabilized with Permeabilization Buffer (Biolegend), incubated with CD68 PE (Y1/82A, Biolegend) and assessed via FACs analyses.

\section{CONFOCAL MICROSCOPY}

BMMs from hCD68-GFP mice were plated in $1.5-\mathrm{mm}$ coverglass chambers $\left(8 \times 10^{4}\right.$ cells/well), treated for $24 \mathrm{~h}$ with vehicle or $\mathrm{rmIL}-10(10 \mathrm{ng} / \mathrm{mL})$ and apBMSCs (stained with CellTracker DeepRed) were added at a $1: 1$ ratio to BMMs for 1-24 $\mathrm{h}$ and fixed with ice-cold methanol for $20 \mathrm{~min}$. Cells were then washed with PBS and covered with ProLong ${ }^{\mathbb{R}}$ Gold antifade reagent with DAPI (Life Technologies). Confocal microscopy images were analyzed using the Leica inverted SP5X confocal microscope system with two-photon film and Leica software (Leica Microsystems). 


\section{MOUSE INFLAMMATION ANTIBODY ARRAY}

BMMs were plated into 6 -well dishes $(1.5 \times 106$ cells/well $)$, then co-cultured with or without apBMSCs at a $1: 1$ ratio in $\alpha$-MEM (0.25\% FBS). Supernatants were collected after $18 \mathrm{~h}$ and proteins were analyzed using the mouse inflammation antibody array $\mathrm{C} 1$ (catalog no. AAM-INF-1-8, RayBiotech, Inc.) per manufacturer's instructions.

\section{TGF- $\beta 1$ ELISA}

Supernatants were collected from BMMs alone, BMMs/apBMSCs or BMMs/apNeutrophils after $18 \mathrm{~h}$ of co-culture. TGF- $\beta 1$ protein levels in culture supernatants were measured with the Quantikine mouse TGF- $\beta 1$ ELISA (RED systems) per manufacturer's instructions. Briefly, acid-activated supernatant samples, standards, and controls were added to anti-mouse TGF- $\beta 1$ antibody pre-coated microplates and incubated at room temperature for $2 \mathrm{~h}$. Wells were washed and incubated with TGF- $\beta 1$ conjugate for $2 \mathrm{~h}$, washed and substrate solution added for $30 \mathrm{~min}$. Stop solution was added and A450 values (corrected with A570) were measured using an EZ Read 400 microplate reader (Biochrom).

\section{QRT-PCR}

Total RNA was isolated from BMMs alone, apBMSCs alone, and BMM/apBMSC co-culture after $18 \mathrm{~h}$ using the Qiagen RNeasy Mini Kit. Reverse transcription PCR was conducted, and the cDNA products were amplified and detected using TaqMan Universal PCR master mix (Applied Biosystems) and TaqMan probes, including mouse Ccl2 (Mm00441242_m1) and mouse Actb (Mm02619580_g1) as an endogenous control. Realtime PCR was analyzed on ABI PRISM 7700 (AppliedBiosystems).

\section{STATISTICAL ANALYSES}

Statistical analyses were performed by unpaired Student's $t$ test to compare two groups or ANOVA to compare three or more groups with a significance of $P<0.05$. Data are presented as mean \pm S.E.

\section{RESULTS}

\section{IL-10 ENHANCES BONE MARROW DERIVED MACROPHAGE ENGULFMENT OF APOPTOTIC BONE MARROW STROMAL CELLS}

To investigate the impact of IL-10 on macrophage-mediated efferocytosis of apoptotic bone cells, bone marrow macrophages (BMMs) were treated with IL-10 and efferocytosis of apoptotic bone marrow stromal cells (apBMSCs) was determined. BMMs $\left(\mathrm{F} 4 / 80-\mathrm{FITC}^{+}\right)$were co-cultured with apBMSCs (DeepRed-APC ${ }^{+}$) and double positive $\left(\mathrm{FITC}^{+} \mathrm{APC}^{+}\right)$cells were quantified using flow cytometric cell sorting, reflecting engulfment (Fig. 1A, left panel). Engulfment was confirmed by ImageStream analysis, identifying double-positive cells as macrophages associated with apBMSCs (Fig. 1A). To assess the effect of various cytokines on efferocytosis, BMMs were treated with IL-10, CCL2, MFG-E8, and sIL-6R for $24 \mathrm{~h}$ and efferocytosis of apBMSCs measured after $1 \mathrm{~h}$ of co-culture. IL-10 enhanced engulfment of apBMSCs by $22 \%$ and in a dosedependent manner (Fig. 1B and C), whereas MCP1, MFG-E8, and sIL-6R did not demonstrate significant changes in efferocytosis at that time point. To determine the optimum IL-10 treatment length, BMMs were treated for 4-48 h with IL-10. BMMs treated for $24 \mathrm{~h}$ with IL-10 displayed increased efferocytosis compared to shorter treatment times (Fig. 1D). Macrophages treated with IL-10 for $24 \mathrm{~h}$ showed enhanced efferocytosis when co-cultured for shorter durations $(0.5-2 \mathrm{~h})$ compared to longer co-cultures $(6 \mathrm{~h})$, suggesting IL-10 increased the rate of efferocytosis (Fig. 1E). IL-10 treatment of BMMs enhanced engulfment of apBMSCs in a time and dose-dependent manner.

\section{IL-10 POLARIZES BONE MARROW DERIVED MACROPHAGES TO M2-LIKE PHENOTYPE WITH ENHANCED EFFEROCYTIC CAPACITY}

Macrophages are described as classically (M1) or alternatively (M2) activated. M2 polarized macrophages are resolving cells with higher efferocytic capacity than M1 macrophages [Xu et al., 2006]. To determine the impact of IL-10 treatment on BMM phenotypes, equal numbers of BMMs were treated with IL-10 for $24 \mathrm{~h}$ and assessed for M1 (F4/80 $\left.{ }^{+} \mathrm{CD}^{+} 6^{+}\right)$and M2 (F4/80 $\left.{ }^{+} \mathrm{CD} 206^{+}\right)$polarization. IL-10 did not affect cell number or viability (Fig. $2 \mathrm{~A})$, mature $\left(\mathrm{CD}^{+} 8^{+}\right)$or $\mathrm{M} 1$ macrophages (Fig. 2B). Treatment with IL-10 significantly increased M2 polarization of BMMs (Fig. 2C). This effect of IL-10 is consistent with previous reports [Goerdt and Orfanos, 1999; Mantovani et al., 2004], however, the impact of IL-10 within the M2 population is unclear. To investigate the role of IL-10 on altering efferocytosis within the $\mathrm{CD}_{206^{+}}$population, efferocytosis of apBMSCs by CD206 ${ }^{+}$ cells was measured. IL-10 increased the percentage of CD206 ${ }^{+}$cells that efferocytosed apBMSCs (Fig. 2D), suggesting that IL-10 not only polarized BMMs to M2 macrophages, but also specifically enhanced their efferocytic ability.

\section{IL-10 INCREASES EFFEROCYTOSIS IN A STAT3 ACTIVATION DEPENDENT MANNER}

IL-10 signals through the JAK/STAT pathway and inhibition of JAK/STAT signaling has been shown to decrease efferocytosis [Soki et al., 2014]. To measure the effect of JAK/STAT pathway inhibition on IL-10 induced efferocytosis, BMMs were pre-treated with the phospho-STAT3 (pSTAT3) inhibitor Stattic prior to IL-10 treatment and efferocytosis measurement. BMMs were isolated from mice which express GFP under the human CD68 promoter to visualize $\mathrm{CD}^{+}{ }^{+}$(mature phagocytic) cells (Fig. 3A). Macrophages harvested from the hCD68-GFP mice displayed increased efferocytosis of apBMSCs following IL-10 treatment as measured by FACs analysis and confocal microscopy (Fig. 3B and C). BMMs pre-treated for $2 \mathrm{~h}$ with Stattic and subsequently treated with IL-10 had reduced efferocytosis of apBMSCs compared to DMSO control treated macrophages (Fig. 3D). Macrophages pre-treated with Stattic followed by vehicle treatment also displayed decreased efferocytosis compared to controls suggesting some baseline efferocytosis in vehicle treated macrophages requires signaling through the JAK/STAT pathway, likely by other cytokine signaling. To investigate the role of JAK/STAT signaling in IL-10 induced M2 polarization, BMMs were treated with Stattic for $2 \mathrm{~h}$ followed by IL-10 treatment and assessed for $\mathrm{CD}_{206}{ }^{+}$(M2 polarization). In vehicle treated BMMs, Stattic did not alter the M2 population; however, Stattic treatment prior to IL-10 treatment significantly reduced the IL-10 mediated M2 shift in macrophage polarization 


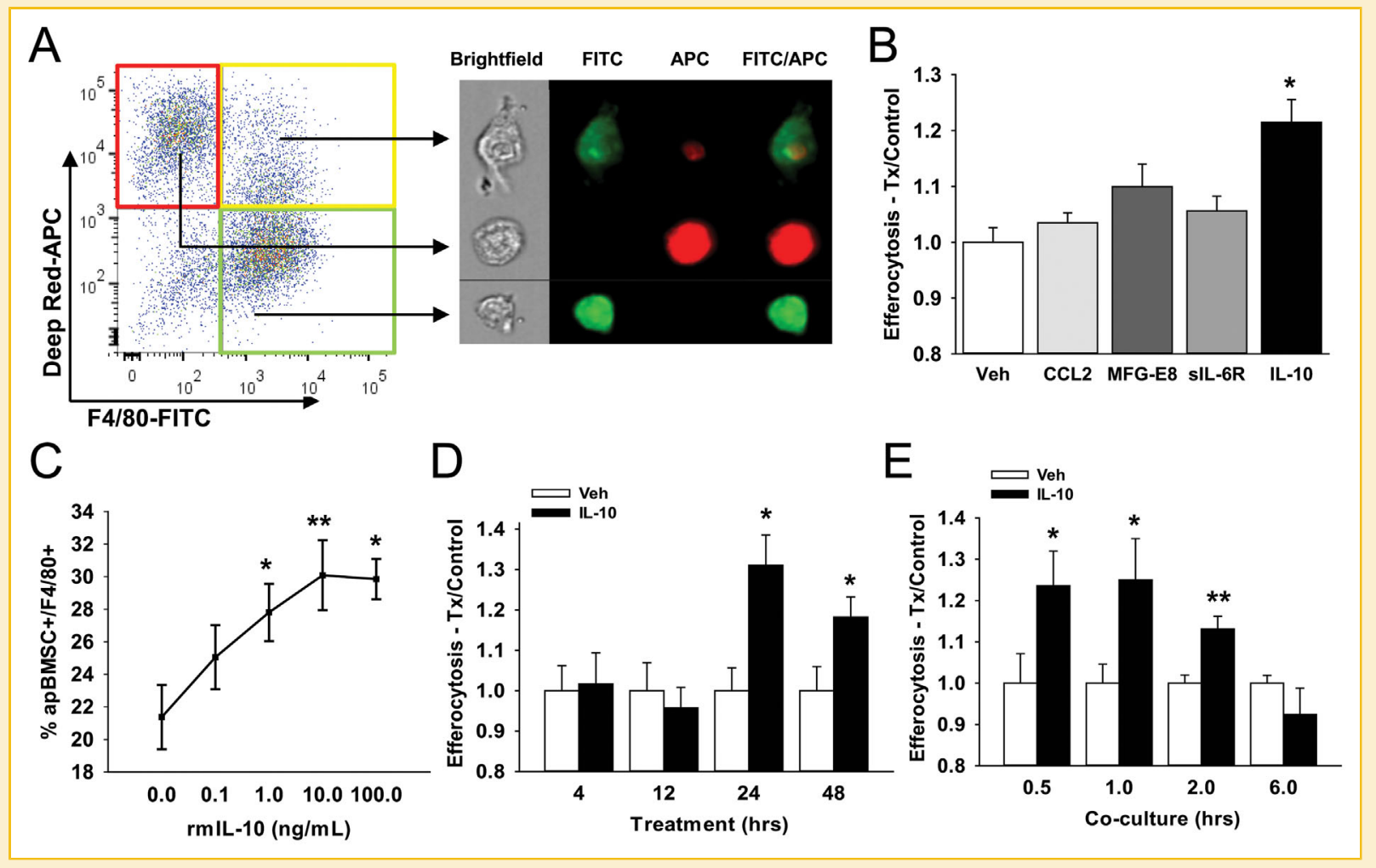

Fig. 1. IL-10 treatment enhanced efferocytosis of apoptotic bone marrow stromal cells (apBMSCs) by bone marrow derived macrophages (BMMs). (A) BMMs were stained for F4/80-FITC and apBMSCs stained with Cell Tracker Deep Red, co-cultured for $1 \mathrm{hr}$ and analyzed via flow cytometry. Representative fluorescence-activated cell sorting (FACs) dot plots (left) indicate macrophages alone (green gate), apBMSCs alone (red gate), or macrophages with internalized apBMSCs (yellow gate). Representative photo from Image Stream which captures single cell images showing either single cells or engulfment. (B) BMMs treated for $24 \mathrm{~h}$ with vehicle, rmlL-10 $(10 \mathrm{ng} / \mathrm{mL}), \mathrm{rmMCP}-1(10 \mathrm{ng} / \mathrm{mL})$, rmMFG-E8 $(500 \mathrm{ng} / \mathrm{mL})$, or sIL-6R $(500 \mathrm{ng} / \mathrm{mL})$ and co-cultured with apBMSCs for $1 \mathrm{~h}$. Efferocytosis was measured as percentage of F4/80 ${ }^{+}$cells which were apBMSC ${ }^{+}$. IL-10 treatment increased efferocytosis compared to vehicle control. (C) BMMs were treated for $24 \mathrm{~h}$ with rmIL-10 $(0.1-100 \mathrm{ng} / \mathrm{mL})$ and co-cultured with apoptotic BMSCs for $1 \mathrm{~h}$. IL-10 (1.0-100 ng/mL) increased efferocytosis of apBMSCs. (D) BMMs were treated with IL-10 (10 ng/mL) for 4-48 h and co-cultured with apBMSCs for $1 \mathrm{~h}$. Treatment of macrophages with rmIL-10 for 24-48 $\mathrm{h}$ induced a larger increase in efferocytosis relative to shorter treatment times. (E) BMMs treated for $24 \mathrm{~h}$ with IL-10 or vehicle control and co-cultured with apoptotic BMSCs for $0.5-12 \mathrm{~h}$. IL-10 increased engulfment of apBMSCs after $0.5-2 \mathrm{~h}$ co-culture. $\mathrm{n}=6 / \mathrm{gp}$, data is mean $\pm \mathrm{SEM}$. ${ }^{*} \boldsymbol{P}<0.05$, ${ }^{* *} \boldsymbol{P}<0.01$ versus vehicle treatment.

(Fig. 3E) suggesting that IL-10 shifts macrophage polarization and enhances efferocytosis of apBMSCs via signaling through JAK/STAT pathway.

\section{MACROPHAGES SECRETE MCP-1/CCL2 AND TGF- $\beta 1$ IN RESPONSE TO APOPTOTIC BONE MARROW STROMAL CELLS}

Macrophages release factors when engulfing apoptotic cells that signal to surrounding cells [Fadok et al., 1998, 2001; Kim et al., 2004]. To determine the response of macrophages to apBMSCs, BMMs were cultured alone or with apBMSCs for $18 \mathrm{~h}$ and supernatants harvested for analysis of secreted proteins. Supernatants harvested from co-culture of BMMs with apBMSCs showed significantly increased secreted CCL2 levels compared to macrophages cultured alone (Fig. 4A). RNA harvested from macrophages co-cultured with apBMSCs also showed increased $\mathrm{ccl} 2$ gene expression (Fig. 4B) compared to BMMs alone or BMMs co-cultured with apoptotic neutrophils. In other tissues, TGF- $\beta 1$ is an important anti-inflammatory cytokine released from macrophages after efferocytosis [Fadok et al., 1998]. Supernatants from macrophages cultured with apBMSCs versus macrophages alone or versus macrophages cultured with apoptotic neutrophils displayed increased total secreted TGF- $\beta 1$ levels (Fig. 4C). Hence, in response to engulfment of apoptotic bone cells, bone marrow macrophages secrete the anti-inflammatory cytokine TGF- $\beta 1$ and chemokine CCL2, which are both important factors related to bone homeostasis.

\section{DISCUSSION}

Patients exhibiting increased inflammation associated with diseases such as rheumatoid arthritis and inflammatory bowel disease are often at a higher risk of generalized osteoporosis and increased fracture risk [Minne et al., 1984; Deodhar and Woolf, 1996; Andreassen et al., 1997]. Chronic inflammation is associated with systemic overproduction of pro-inflammatory mediators, which are thought to play a role in decreasing bone formation [Pfeilschifter et al., 1987]. Periodontal disease activates macrophages and triggers systemic inflammation of the vasculature and atherosclerotic signs [Miyajima et al., 2014]. Macrophages are immune cells which 


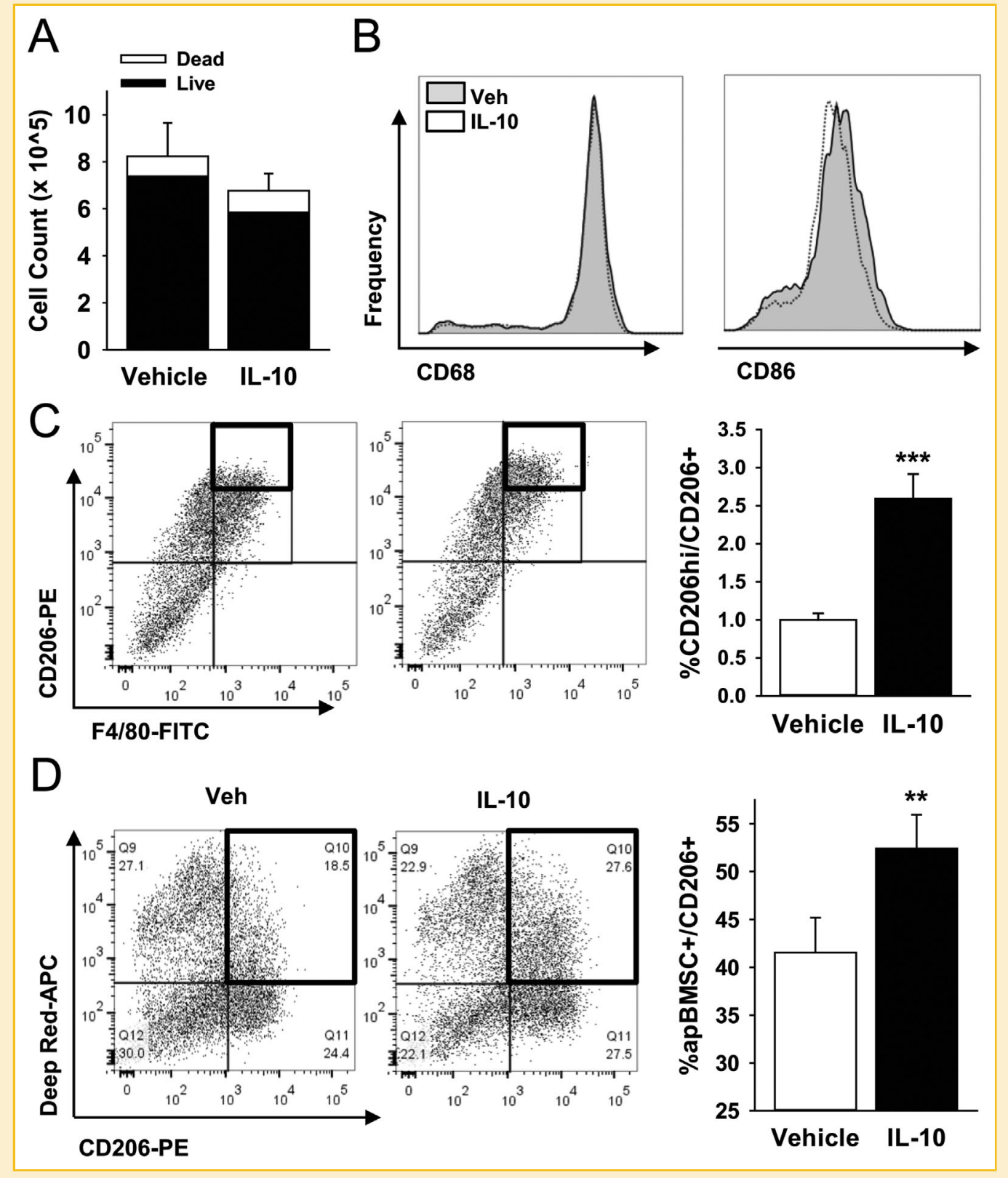

Fig. 2. IL-10 polarizes BMMs to alternatively activated (M2) phenotype. (A) BMMs were treated with $\mathrm{rmlL}-10(10 \mathrm{ng} / \mathrm{mL})$ for $24 \mathrm{~h}$ and enumerated. IL-10 did not change cell number compared to vehicle controls or viability, (B) BMMs treated for $24 \mathrm{~h}$ with rmIL-10 were analyzed via FACs for phagocytic cells (CD68) and M1 macrophage phenotype (CD86). No significant changes were observed in these cell populations with IL-10 treatment. (C) BMMs treated with IL-10 were analyzed via FACs analysis for alternatively activated M2 macrophages (CD206). IL-10 treatment significantly increased the CD206 ${ }^{\text {hi }}$ population per CD206 ${ }^{+}$. (D) BMMs treated with IL-10 showed enhanced efferocytosis of apBMSCs within the $\mathrm{CD}_{206}{ }^{+}$population after $1 \mathrm{~h}$ co-culture as evidences by increased $\mathrm{CD} 206^{+}$apBMSCs ${ }^{+}$double positive cells. $\mathrm{n}=6 / \mathrm{gp}$, data is mean $\pm \mathrm{SEM}$. ${ }^{* *} P<0.01$, *** $P<0.001$ versus vehicle treatment.

mediate pro-inflammatory or anti-inflammatory conditions yet little is known about their role in the basic cell biology of bone.

Macrophages have recently become of interest in the bone field due to their intimate association with the bone forming unit and contributions to osteoblast function [Chang et al., 2008; Alexander et al., 2011; Cho et al., 2014]. It is hypothesized that the antiinflammatory function of macrophages and the clearance of apoptotic cells may contribute to bone turnover and regeneration
[McCauley et al., 2014]. In humans, billions of cells die daily which is unappreciated by current methods used to detect apoptotic cells from in vivo tissue samples. This is due to the rapid and effective clearance of apoptotic cells, termed efferocytosis [Ravichandran and Lorenz, 2007]. Macrophages are vital mediators of cellular turnover, maintenance of extracellular matrix homeostasis and modulation, and resolution of inflammation [Hasturk et al., 2012]. Understanding their mechanisms of action as they relate to bone remodeling 


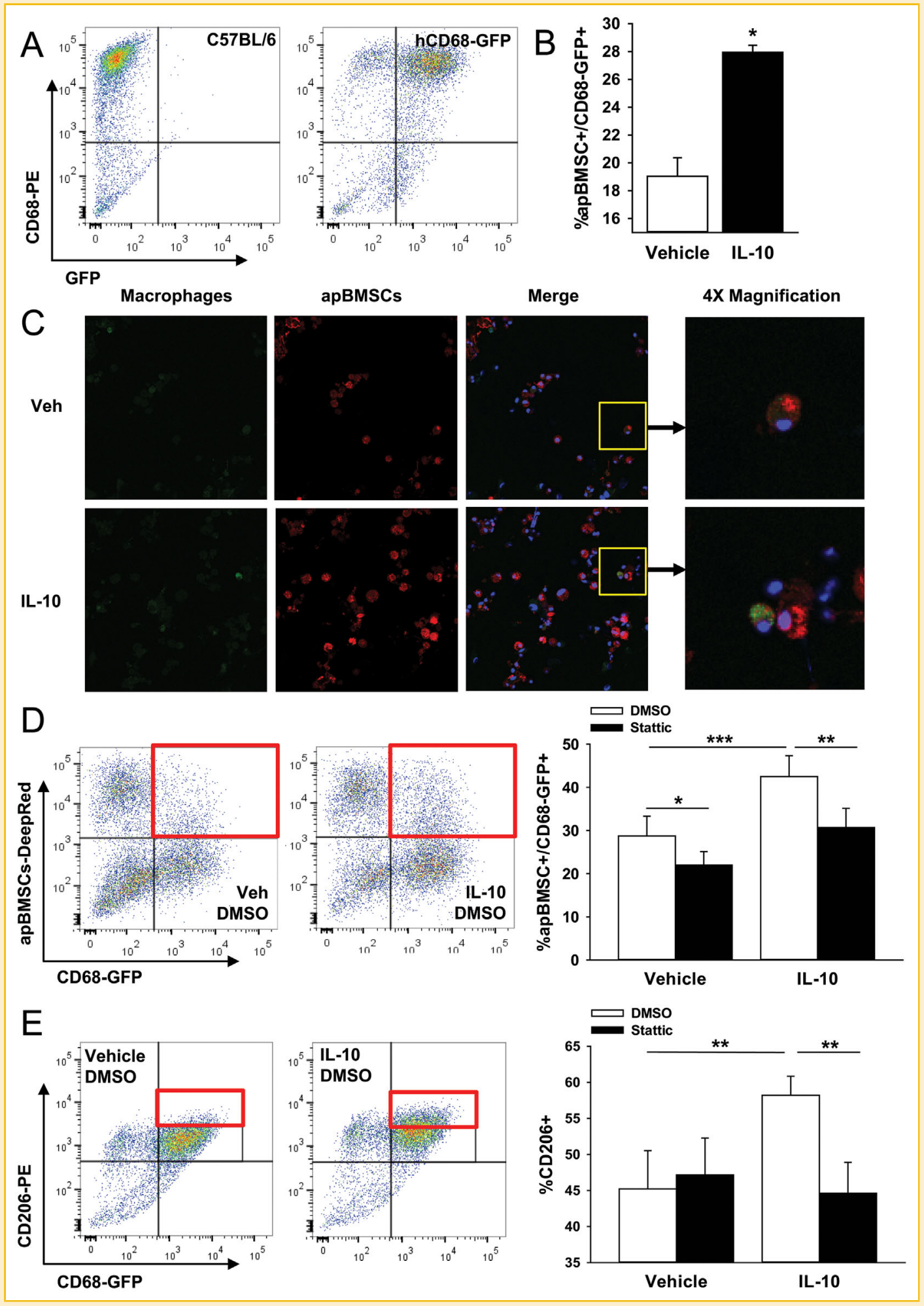

Fig. 3. Stat3 inhibition diminished IL-10 effect. (A) BM cells were harvested from 4- to 8-week old hCD68-GFP mice, enriched to macrophages with M-CSF then analyzed for GFP retention. FACs analysis showed that the majority of CD68-PE+ cells were also GFP+, indicating GFP was retained during in vitro culture. (B) hCD68-GFP BMMs were treated with IL-10 for $24 \mathrm{~h}$ and efferocytosis of apBMSCs harvested from C57BL/6J analyzed via FACs analysis. IL-10 significantly increased the percentage of GFP ${ }^{+}$cells which were also positive for apBMSCs (Deep Red). (C) hCD68-GFP BMMs were treated with IL-10 for $24 \mathrm{~h}$ and efferocytosis of apBMSCs analyzed via confocal microscopy. (D) hCD68-GFP BMMs were treated with Stat3 inhibitor (Stattic, $6.25 \mu \mathrm{M}$ ) or DMSO for $2 \mathrm{~h}$ followed by IL-10 or vehicle treatment for $24 \mathrm{~h}$. Efferocytosis of apBMSCs was analyzed via FACs analysis for double positive GFP/apBMSCs cells (representative dot plots on left). Stat3 inhibitor decreased efferocytosis of apBMSCs in vehicle and IL-10 treated BMMs. (E) Alternatively activated macrophage phenotype (CD206-PE) was increased in IL-10 treated BMMs and the effect was diminished with Stattic pre-treatment. $\mathrm{n}=6$ /group, data is mean \pm SEM. ${ }^{*} \boldsymbol{P}<0.05,{ }^{* *} \boldsymbol{P}<0.01,{ }^{* * *} \boldsymbol{P}<0.001$ versus vehicle treatment. 


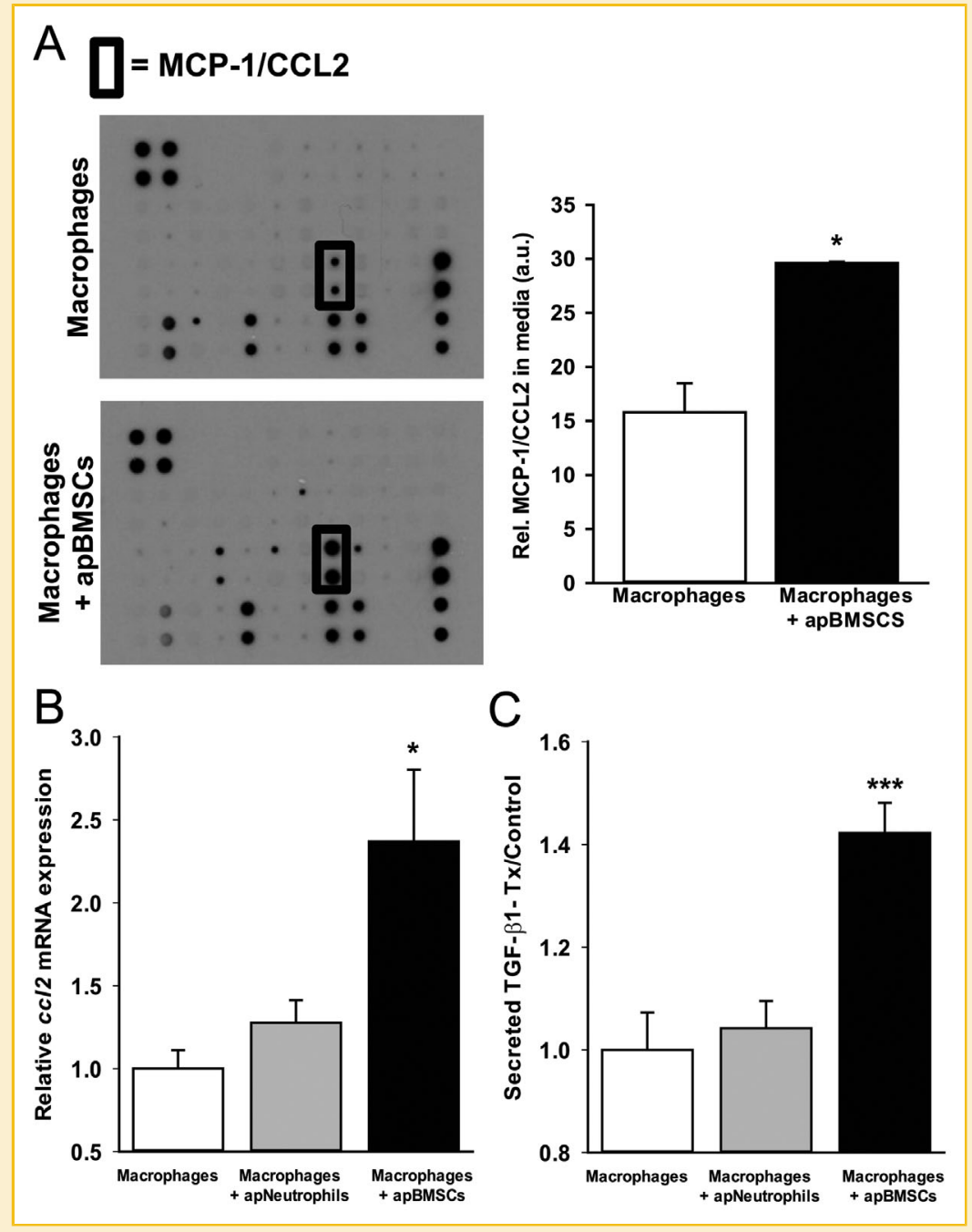

Fig. 4. Macrophage efferocytosis induces CCL2 and TGF- $\beta 1$ secretion. (A) Supernatants were harvested from BMMs, apBMSCs or co-culture of BMMs with apBMSCs for $18 \mathrm{~h}$ and analyzed for secreted proteins using a murine inflammatory cytokine array. Co-culture of BMMs with apBMSCs showed significantly increased secreted CCL2 compared to BMMs or apBMSCs cultured alone (arbitrary units, a.u.). (B) RNA was isolated from BMMs alone or co-cultured with apBMSCs or apoptotic neutrophils and QRT-PCR performed for $c c / 2$ mRNA. $C c / 2$ gene expression was increased in co-culture of BMMs with apBMSCs compared to BMMs alone. (C) ELISA for total TGF- $\beta 1$ levels in the supernatants showed increased secreted TGF- $\beta 1$ after $18 \mathrm{~h}$ of co-culture of BMMs with apBMSCs compared to BMMs, $\mathrm{n}=6 / \mathrm{gp}$ data is mean $\pm \mathrm{SEM}$. ${ }^{*} \boldsymbol{P}<0.05$, ${ }^{* * *} \boldsymbol{P}<0.001$ versus macrophages cultured alone.

provides a basis for the prevention and treatment of chronic inflammatory conditions.

Anti-inflammatory cytokines such as IL-4 and IL-10 released by immune cells have been shown to increase human peripheral blood macrophage engulfment of non-bone associated apoptotic cells [Ogden et al., 2005; Lingnau et al., 2007]. IL-10 contributes to bone turnover by regulating osteoclastogenesis and IL-10 deficient mice display osteopenia [Dresner-Pollak et al., 2004; Fujioka et al., 2015], but the role of IL-10-associated efferocytosis by bone marrow macrophages is largely unknown. To study mechanisms of efferocytosis in bone marrow derived macrophages, a series of in vitro experiments were performed to determine how bone macrophages respond to apoptotic bone marrow stromal cells and what factors may influence the rate of engulfment. IL-10 treated macrophages displayed enhanced clearance of apoptotic bone marrow stromal cells. The effect of IL-10 on bone marrow macrophages was in part due to polarization of macrophages toward the pro-resolving M2 phenotype. While there was a significant increase in M2 macrophages, the increase was not the only contributing factor to an increase in efferocytosis. The CD206 ${ }^{+}$ M2 macrophages displayed increased efferocytosis of apoptotic bone cells after treatment with IL-10 compared to vehicle treated macrophages. This suggests that not only does IL-10 polarize macrophages to M2, but it also enhances the M2 macrophage efferocytic capacity.

The effect of IL-10 on efferocytosis of apoptotic lymphocytes and neutrophils has been measured, and downstream signaling of IL-10 has been identified [Ogden et al., 2005; Lingnau et al., 2007]. IL-10 signals through the JAK/STAT pathway, in which STAT3 is phosphorylated, dimerizes, and translocates to the nucleus to 


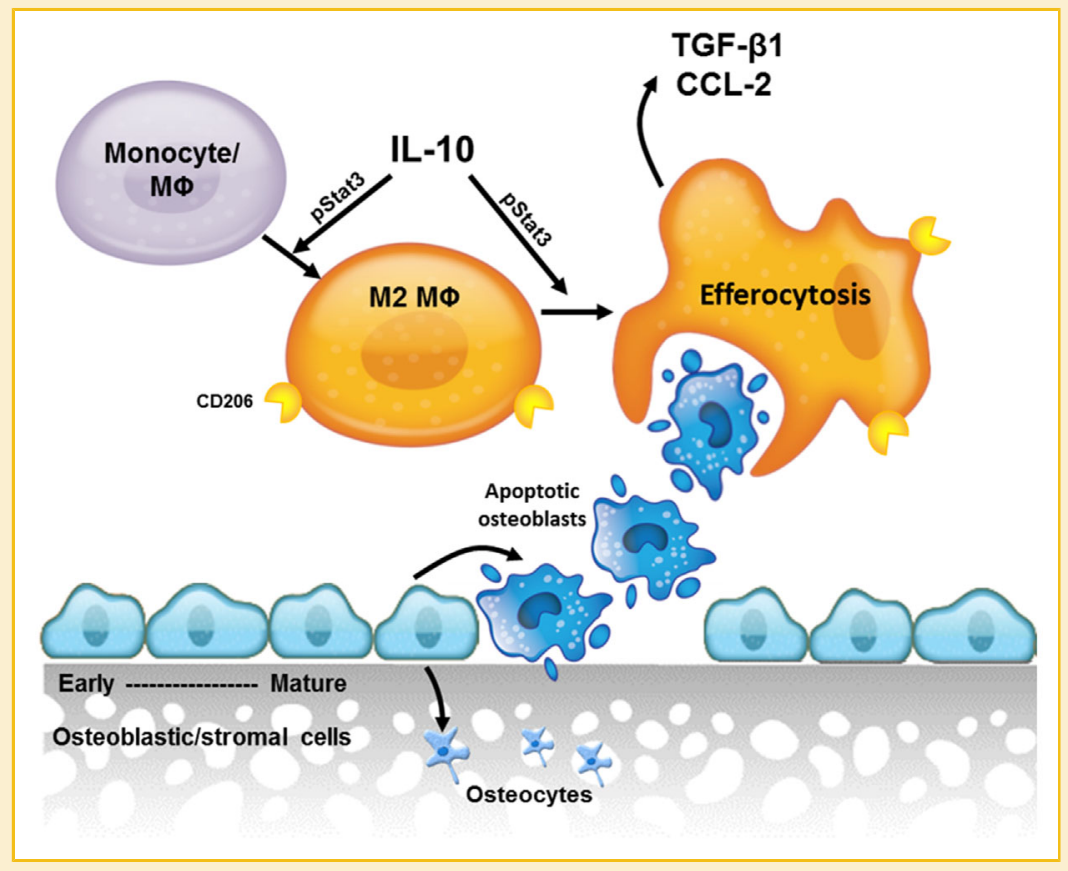

Fig. 5. Model. IL-10 promotes M2 macrophage polarization and enhances clearance of apoptotic bone marrow stromal cells in a STAT3-phosphorylation dependent manner. Clearance of apoptotic cells results in release of anti-inflammatory and osteoinductive factors (TGF- $\beta 1$ and CCL2) which aid in the recruitment of monocytes and osteoclast precursors.

regulate transcription of various genes [Liu et al., 1994; Donnelly et al., 1999; Lang et al., 2002]. To validate the downstream events of IL-10 signaling, macrophages were treated with an inhibitor of STAT3 phosphorylation (Stattic) prior to IL-10 treatment. Efferocytosis of apoptotic bone cells was decreased with Stattic treatment, demonstrating that the IL-10 effect occurs in a pSTAT3-dependent manner. Previous studies have also indicated that inhibition of STAT3 phosphorlyation decreases MFG-E8 mediated phagocytosis of apoptotic mimicry beads [Soki et al., 2014].

Following IL-10 activation of macrophages and efferocytosis of apoptotic bone cells, proteins released by macrophages signal to surrounding cells. How macrophages respond to apoptotic bone cells may indicate what effects efferocytosis have on bone modeling and remodeling. The identity of the apoptotic cell, bone marrow stromal cells in this case, contributes to the profile of genes upregulated in macrophages partaking in efferocytosis. In the present study, bone marrow derived macrophages increased secretion of monocyte chemoattractant protein-1/CC chemokine ligand 2 (MCP-1/CCL2) after co-culture with apoptotic bone cells. CCL2 is associated with monocyte/osteoclast precursor recruitment and enhances osteoclastogenesis [Li et al., 2007]. These data suggest that CCL2 secreted from macrophages engulfing apoptotic bone cells could aid in the recruitment of monocytes to enhance bone turnover by increasing osteoclasts and bone macrophages.

TGF- $\beta 1$ was also found to be increased after efferocytosis of apBMSCs. Previous studies using human peripheral blood macrophages showed increased TGF- $\beta 1$ levels following efferocytosis of apoptotic human derived neutrophils [Fadok et al., 1998]. The release of TGF- $\beta 1$ following apoptotic cell engulfment is often credited with an anti-inflammatory function. Interestingly, in the context of bone, TGF- $\beta 1$ enhances the recruitment of mesenchymal stem cells which are precursors to osteoblastic cells [Tang et al., 2009; Koh et al., 2011]. The secretion of TGF- $\beta 1$ in response to apoptotic bone cells may contribute to repopulating the bone forming units. Other osteogenic factors such as BMP-2 and osteopontin have been shown to be released from M2 macrophages, but it is unclear how the protein levels change during the process of efferocytosis [Takahashi et al., 2004; Honda et al., 2006].

Macrophages are crucial regulators of bone turnover in steady state bone remodeling and wound healing [Chang et al., 2008; Alexander et al., 2011]. A better understanding of the role of these macrophages is important in developing ways to utilize macrophages in therapeutic interventions for osteoporosis, fracture healing, periodontal wound healing, and osteonecrosis. These data demonstrate one mechanism by which macrophages efferocytose apoptotic bone cells and their subsequent release of factors important in bone remodeling (Fig. 5). Future work to determine the association of efferocytosis and bone turnover is important to better understand the mechanisms by which macrophages contribute to bone modeling and remodeling.

\section{ACKNOWLEDGMENTS}

The work was supported by the National Institutes of Health, National Institute of Diabetes, and Digestive and Kidney Diseases [R01DK053904]; and the National Institutes of Health, National Institute of Dental, and Craniofacial Research [F30DE025154, T32DE007057]. The authors would like to thank Dr. Ajay Chawla 
from University of California San Francisco for providing the hCD68-GFP reporter mice. The authors would like to thank Dr. Benjamin P. Sinder from the University of Michigan School of Dentistry for his assistance with the cytokine array and experimental design discussions, Sasha Meschinchi from the Microscopy \&t Image Analysis Laboratory for assistance with confocal imaging, and Dave Adams and Michael Pihalja for help with ImageStream collection and analysis.

\section{REFERENCES}

Alexander KA, Chang MK, Maylin ER, Kohler T, Muller R, Wu AC, Van Rooijen N, Sweet MJ, Hume DA, Raggatt LJ, Pettit AR. 2011. Osteal macrophages promote in vivo intramembranous bone healing in a mouse tibial injury model. J Bone Miner Res 26:1517-1532.

Andreassen H, Rungby J, Dahlerup JF, Mosekilde L. 1997. Inflammatory bowel disease and osteoporosis. Scand J Gastroenterol 32:1247-1255.

Bystrom J, Evans I, Newson J, Stables M, Toor I, van Rooijen N, Crawford M, Colville-Nash P, Farrow S, Gilroy DW. 2008. Resolution-phase macrophages possess a unique inflammatory phenotype that is controlled by cAMP. Blood 112:4117-4127.

Champagne CM, Takebe J, Offenbacher S, Cooper LF. 2002. Macrophage cell lines produce osteoinductive signals that include bone morphogenetic protein-2. Bone 30:26-31.

Chang MK, Raggatt LJ, Alexander KA, Kuliwaba JS, Fazzalari NL, Schroder K, Maylin ER, Ripoll VM, Hume DA, Pettit AR. 2008. Osteal tissue macrophages are intercalated throughout human and mouse bone lining tissues and regulate osteoblast function in vitro and in vivo. J Immunol 181:1232-1244.

Cho SW, Soki FN, Koh AJ, Eber MR, Entezami P, Park SI, van Rooijen N, McCauley LK. 2014. Osteal macrophages support physiologic skeletal remodeling and anabolic actions of parathyroid hormone in bone. Proc Natl Acad Sci USA 111:1545-1550.

Deodhar AA, Woolf AD. 1996. Bone mass measurement and bone metabolism in rheumatoid arthritis: A review. Br J Rheumatol 35:309-322.

Donnelly RP, Dickensheets H, Finbloom DS. 1999. The interleukin-10 signal transduction pathway and regulation of gene expression in mononuclear phagocytes. J Interferon Cytokine Res 19:563-573.

Dresner-Pollak R, Gelb N, Rachmilewitz D, Karmeli F, Weinreb M. 2004. Interleukin 10-deficient mice develop osteopenia, decreased bone formation, and mechanical fragility of long bones. Gastroenterology 127:792-801.

Fadok VA, Bratton DL, Guthrie L, Henson PM. 2001. Differential effects of apoptotic versus lysed cells on macrophage production of cytokines: Role of proteases. J Immunol 166:6847-6854.

Fadok VA, Bratton DL, Konowal A, Freed PW, Westcott JY, Henson PM. 1998. Macrophages that have ingested apoptotic cells in vitro inhibit proinflammatory cytokine production through autocrine/paracrine mechanisms involving TGF-beta, PGE2, and PAF. J Clin Invest 101:890-898.

Fujioka K, Kishida T, Ejima A, Yamamoto K, Fujii W, Murakami K, Seno T, Yamamoto A, Kohno M, Oda R, Yamamoto T, Fujiwara H, Kawahito Y, Mazda 0. 2015. Inhibition of osteoclastogenesis by osteoblast-like cells genetically engineered to produce interleukin-10. Biochem Biophys Res Commun 456:785-791.

Goerdt S, Orfanos CE. 1999. Other functions, other genes: Alternative activation of antigen-presenting cells. Immunity 10:137-142.

Hasturk H, Kantarci A, Van Dyke TE. 2012. Oral inflammatory diseases and systemic inflammation: Role of the macrophage. Front Immunol 3:118.

Honda Y, Anada T, Kamakura S, Nakamura M, Sugawara S, Suzuki 0. 2006. Elevated extracellular calcium stimulates secretion of bone morphogenetic protein 2 by a macrophage cell line. Biochem Biophys Res Commun 345:1155-1160.
Iqbal AJ, McNeill E, Kapellos TS, Regan-Komito D, Norman S, Burd S, Smart N, Machemer DE, Stylianou E, McShane H, Channon KM, Chawla A, Greaves DR. 2014. Human CD68 promoter GFP transgenic mice allow analysis of monocyte to macrophage differentiation in vivo. Blood 124: e33-e44.

Jilka RL, Weinstein RS, Bellido T, Parfitt AM, Manolagas SC. 1998. Osteoblast programmed cell death (apoptosis): Modulation by growth factors and cytokines. J Bone Miner Res 13:793-802.

Kim S, Elkon KB, Ma X. 2004. Transcriptional suppression of interleukin-12 gene expression following phagocytosis of apoptotic cells. Immunity 21:643-653.

Koh AJ, Novince CM, Li X, Wang T, Taichman RS, McCauley LK. 2011. An irradiation-altered bone marrow microenvironment impacts anabolic actions of PTH. Endocrinology 152:4525-4536.

Lang R, Patel D, Morris JJ, Rutschman RL, Murray PJ. 2002. Shaping gene expression in activated and resting primary macrophages by IL- 10 . J Immunol 169:2253-2263.

Li X, Qin L, Bergenstock M, Bevelock LM, Novack DV, Partridge NC. 2007. Parathyroid hormone stimulates osteoblastic expression of MCP-1 to recruit and increase the fusion of pre/osteoclasts. J Biol Chem 282:33098-33106.

Lingnau M, Hoflich C, Volk HD, Sabat R, Docke WD. 2007. Interleukin-10 enhances the CD14-dependent phagocytosis of bacteria and apoptotic cells by human monocytes. Hum Immunol 68:730-738.

Liu Y, Wei SH, Ho AS, de Waal Malefyt R, Moore KW. 1994. Expression cloning and characterization of a human IL-10 receptor. J Immunol 152:1821-1829.

Mantovani A, Sica A, Sozzani S, Allavena P, Vecchi A, Locati M. 2004. The chemokine system in diverse forms of macrophage activation and polarization. Trends Immunol 25:677-686.

McCauley LK, Dalli J, Koh AJ, Chiang N, Serhan CN. 2014. Cutting Edge: Parathyroid Hormone Facilitates Macrophage Efferocytosis in Bone Marrow via Proresolving Mediators Resolvin D1 and Resolvin D2. J Immunol 193:26-29.

Minne HW, Pfeilschifter J, Scharla S, Mutschelknauss S, Schwarz A, Krempien B, Ziegler R. 1984. Inflammation-mediated osteopenia in the rat: A new animal model for pathological loss of bone mass. Endocrinology 115:50-54.

Miyajima S, Naruse K, Kobayashi Y, Nakamura N, Nishikawa T, Adachi K, Suzuki Y, Kikuchi T, Mitani A, Mizutani M, Ohno N, Noguchi T, Matsubara T. 2014. Periodontitis-activated monocytes/macrophages cause aortic inflammation. Sci Rep 4:5171.

Moretti S, Bartolommei L, Galosi C, Renga G, Oikonomou V, Zamparini F, Ricci G, Borghi M, Puccetti M, Piobbico D, Eramo S, Conti C, Lomurno G, Bartoli A, Napolioni V, Romani L. 2015. Fine-tuning of Th17 Cytokines in Periodontal Disease by IL-10. J Dent Res 94:1267-1275.

Ogden CA, Pound JD, Batth BK, Owens S, Johannessen I, Wood K, Gregory CD. 2005. Enhanced apoptotic cell clearance capacity and B cell survival factor production by IL-10-activated macrophages: Implications for Burkitt's lymphoma. J Immunol 174:3015-3023.

Pfeilschifter J, Wuster C, Vogel M, Enderes B, Ziegler R, Minne HW. 1987. Inflammation-mediated osteopenia (IMO) during acute inflammation in rats is due to a transient inhibition of bone formation. Calcif Tissue Int 41:321-325.

Ravichandran KS, Lorenz U. 2007. Engulfment of apoptotic cells: Signals for a good meal. Nat Rev Immunol 7:964-974.

Sinder BP, Pettit AR, McCauley LK. 2015. Macrophages: Their emerging roles in bone. J Bone Miner Res 30:2140-2149.

Soki FN, Koh AJ, Jones JD, Kim YW, Dai J, Keller ET, Pienta KJ, Atabai K, Roca H, McCauley LK. 2014. Polarization of prostate cancer associated macrophages is induced by milk-fat globule-EGF Factor 8 (MFG-E8) mediated efferocytosis. J Biol Chem 289:24560-24572. 
Swamydas M, Lionakis MS. 2013. Isolation, purification and labeling of mouse bone marrow neutrophils for functional studies and adoptive transfer experiments. J Vis Exp 77:e50586.

Takahashi F, Takahashi K, Shimizu K, Cui R, Tada N, Takahashi H, Soma S, Yoshioka M, Fukuchi Y. 2004. Osteopontin is strongly expressed by alveolar macrophages in the lungs of acute respiratory distress syndrome. Lung 182:173-185.

Tang Y, Wu X, Lei W, Pang L, Wan C, Shi Z, Zhao L, Nagy TR, Peng X, Hu J, Feng X, Van Hul W, Wan M, Cao X. 2009. TGF-beta1-induced migration of bone mesenchymal stem cells couples bone resorption with formation. Nat Med 15:757-765.
Voll RE, Herrmann M, Roth EA, Stach C, Kalden JR, Girkontaite I. 1997. Immunosuppressive effects of apoptotic cells. Nature 390:350351.

Xu LX, Kukita T, Kukita A, Otsuka T, Niho Y, Iijima T. 1995. Interleukin-10 selectively inhibits osteoclastogenesis by inhibiting differentiation of osteoclast progenitors into preosteoclast-like cells in rat bone marrow culture system. J Cell Physiol 165:624-629.

Xu W, Roos A, Schlagwein N, Woltman AM, Daha MR, van Kooten C. 2006. IL-10-producing macrophages preferentially clear early apoptotic cells. Blood 107:4930-4937. 